\title{
The Pterional Approach and Extradural Anterior Clinoidectomy to Clip Paraclinoid Aneurysms
}

\author{
Jung Soo Kim, Sun-il Lee, Kyoung Dong Jeon, Byeong Sam Choi \\ Department of Neurosurgery, Haeundae Paik Hospital, Inje University Colleage of Medicine, Busan, Korea
}

Objective : The surgical clipping of paraclinoid segment internal carotid artery aneurysms is considered difficult because of the complex anatomical location and important neighboring structures. Our experiences of pterional craniotomy and extradural anterior clinoidectomy (EAC) to clip paraclinoid aneurysms are reported herein.

Methods : We present two patients with paraclinoid aneurysms who underwent surgical clipping using pterional craniotomy and EAC. The clinical results and operative techniques were reviewed from the patients' medical records.

Results : EAC improves the surgical field in the suprasellar and periclinoid regions. Clinically, a good outcome was obtained in both cases. No surgical complications directly resulting from the EAC were observed.

Conclusion : Favorable surgical results can be obtained with pterional craniotomy and EAC for the clipping of paraclinoid aneurysms. EAC is advocated for the clipping of paraclinoid aneurysms.

Keywords Paraclinoid aneurysm, Extradural, Anterior clinoidectomy, Pterional craniotomy

J Cerebrovasc Endovasc Neurosurg.

2013 September; 15(3):260-266

Received : 24 May 2013

Revised : 15 July 2013

Accepted : 13 August 2013

Correspondence to Sun-il Lee

Department of Neurosurgery, Haeundae

Paik Hospital, Inje University College of

Medicine, 1435, Jwa 4-dong, Haeundae-gu,

Busan 612-862, Korea

Tel : 82-51-797-3805

Fax : 82-51-797-0841

E-mail : nssunlee@inje.ac.kr

This is an Open Access article distributed under the terms of the Creative Commons Attribution NonCommercial License (http://creativecommons.org/licenses/by-nc/3.0) which permits unrestricted noncommercial use, distribution, and reproduction in any medium, provided the original work is properly cited.

\section{INTRODUCTION}

The anterior clinoid process (ACP) is the apex portion of the lesser wing of the sphenoid bone. ACP's anatomical location is important for its relation with neighboring structures including optic nerves, internal carotid artery (ICA), and other neurovascular structures. ${ }^{7)}$ Anterior clinoidectomy (AC) is an essential technique that is of utmost importance for clipping aneurysms involving the paraclinoid region. Dolenc introduced the extradural AC (EAC) and opening of the optic sheath in 1985. ${ }^{2}$ We can retract the brain minimally to remove the whole ACP since the dura provides a natural barrier to preserve the neurovascular structures from drilling, and separates the subarachnoid space from the bone dusts. We Reports the current techniques for EAC with clipping of paraclinoid aneurysm.

\section{MATERIALS AND METHODS}

Two patients with paraclinoid aneurysm (1 ruptured, 1 unruptured) were treated, and it was found that pterional craniotomy and EAC are more suitable than coil embolization for the clipping of aneurysm. The patients were females aged 42 and 46 . One patient was diagnosed using magnetic resonance imaging (MRI) due to a visual field defect, and the other patient was diagnosed using computed tomography (CT) due to the presence of intracerebral hemorrhage with ruptured aneurysm. 


\section{SURGICAL TECHNIQUE}

\section{Pterional approach with interfacial muscle dissection}

The ipsilateral pterional approach is often used for treating paraclinoid aneurysms. The patient's head is elevated 10-30 degrees and rotated 30-45 degrees to the contralateral side and fixed onto the Mayfield head fixator. The ipsilateral neck is draped so that the carotid artery can be compressed manually in an emergency. The cervical carotid artery is usually not exposed to allow temporary occlusion.

A curvilinear skin incision starts at the zygomatic arch $1 \mathrm{~cm}$ anterior to the tragus and curves to the midline, just behind the hairline. Interfacial temporalis muscle dissection is often done for more temporal-base exposure, and the temporal branch of the facial nerve is preserved.

Frontotemporal craniotomy is done using 2 or more burr holes. The craniotomy follows the temporalis incision posteriorly then arcs anteromedially to the supraorbital norch, and inferiorly to the bottom of the orbital and temporal base. The drill is used to take off the lesser wing of the sphenoid medially to the superior orbital fissure, with a flat surface over the orbit connecting the anterior and middle cranial fossae (Fig. 1 , blue area).

\section{Extradural removal of the ACP: Periosteal dural dissection}

The extradural removal of the ACP is accomplished using a microscope after the outer two-thirds of sphenoid ridge is drilled. The dura on the orbital roof is softly detached with a dissector. This stage yields mobility for the neurovascular structures of the superior orbital fissure (SOF) and exposes the dural fold at the top of the SOF, where the meningo-orbital band (MOB) is located. The SOF is an inset of the external dural layer of the temporal fossa, which runs through the superior orbital fissure and conjoins the periorbita at the level of the sphenoparietal sinus (Fig. 2A). The $\mathrm{ACP}$ is drilled partially, and the MOB that runs the bone contour SOF and attaches to the periorbita opened. The dura mater of the frontal and temporal fossa is divided to expose the MOB superomedially and the SOF inferolaterally (Fig. 2B). The MOB and the junction of periorbital dura mater are opened at the lateral wall of the cavernous sinus. The MOB is mutilated using a pair of sharp scissors or an 11 blade. The inner and outer dura layer of the lateral cavernous sinus is carefully split off to expose all the dimensions of the ACP.

\section{Extradural removal of the ACP: Drilling of the less- er wing of sphenoid bone}

The posterior half of the roof and lateral wall of the orbit and sphenoid ridge covering the SOF are resected until the orbital portion of the optic nerve is clearly identified. The ACP drilling is done with a 3 $\mathrm{mm}$ or less diamond drill under continuous irrigation to prevent thermal injury to the neurovascular structures. The drilling starts from the lateral side of the ACP to medial part (Fig. 1, red area). The oculomotor nerve passes the inferolateral side of the ACP.

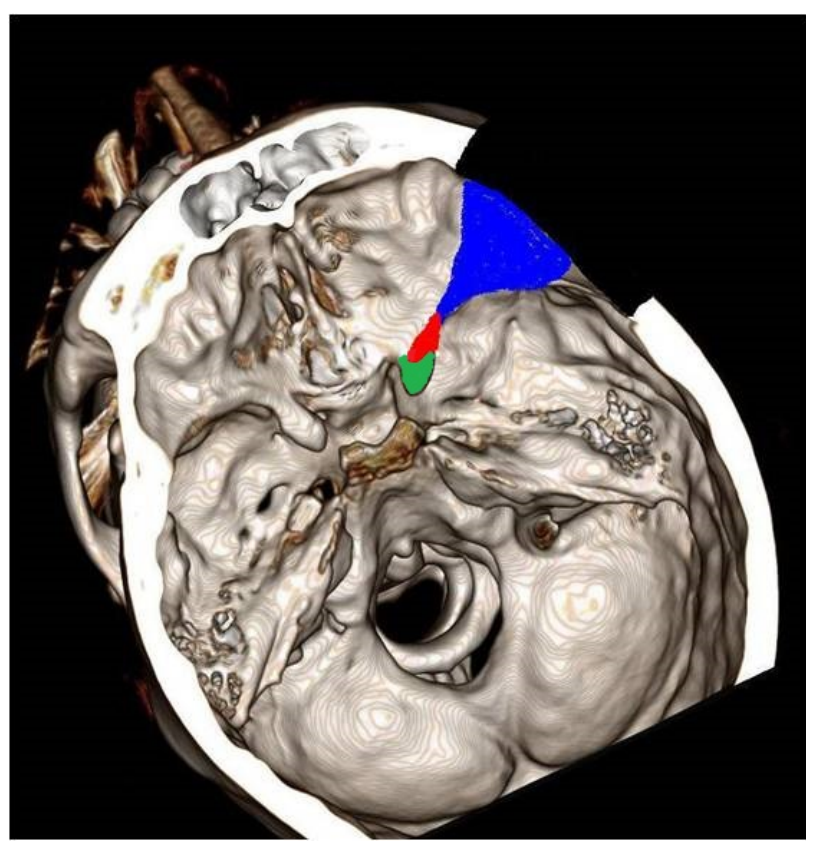

Fig. 1. Schematic diagram: head position for pterional craniotomy; after pterional craniotomy and removal of the sphenoid lesser wing (Blue area), Schematic diagram of the lesser wing of the sphenoid and of the optic-canal-roof drilling (Red area), Schematic diagram of the anterior clinoid process's centrally shelled or hollowed-out area (Green area). 

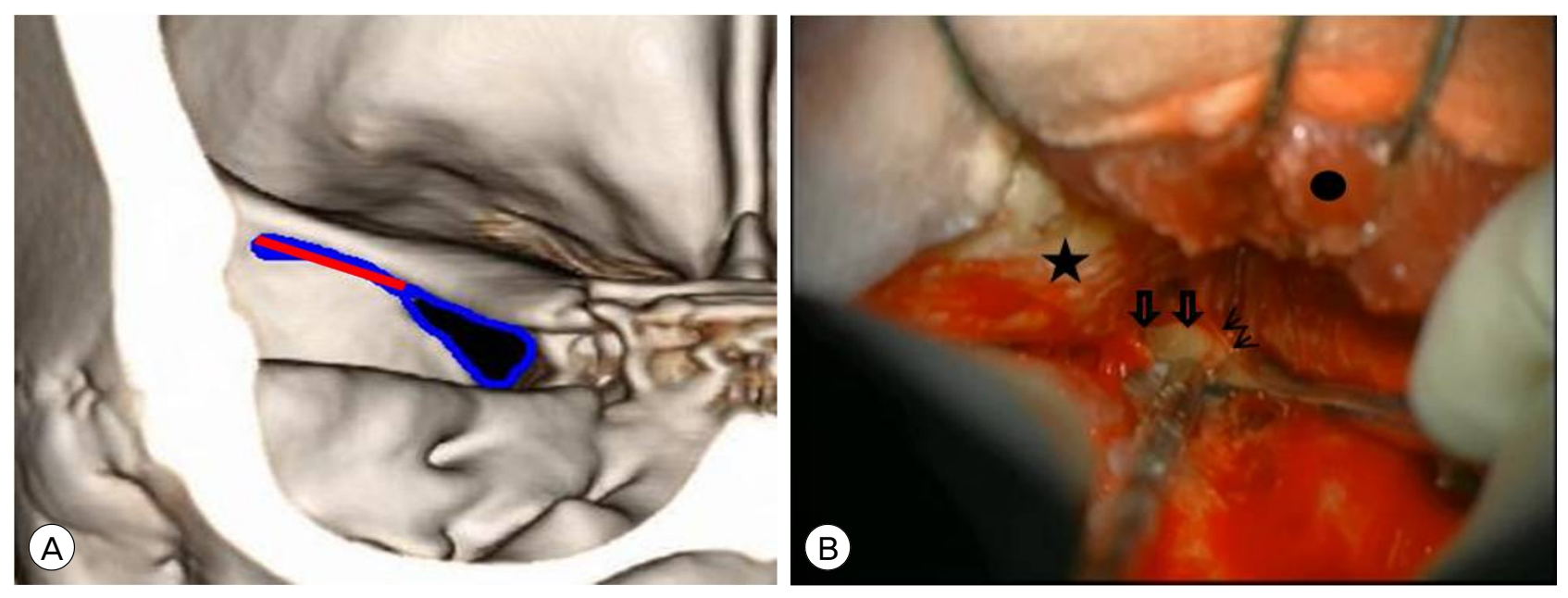

Fig. 2. Schematic diagram of the middle skull base posterior to anterior view: (A) superior orbital fissure (Blue line) and meningoorbital band (Red line). (B) Intraoperative photography: The temporalis muscle (Dark circle) is retracted red inferiorly. The meningoorbital band (MOB) and outer dura layer of the lateral wall of the cavernous sinus is peeled. The outer two-thirds of sphenoid ridge (Asterisk) is drilled away. The entire lesser wing of the sphenoid is seen (Open arrow). The dura mater of the frontal and temporal fossa is divided to expose the MOB (Beneath the suction tip) superomedially and the dural fold of the superior orbital fissure (Closed arrow) inferolaterally. The dark circle is temporalis muscle and muscle retractor.

Attention is required while drilling the inferolateral side of the ACP.

\section{Removal of the roof of the optic canal and optic strut}

The apex of the ACP is removed using a diamond burr with constant irrigation inside the cancellous bone to lateral cortical bone. The cortical bone of the $\mathrm{ACP}$ is remained like a thinned onion shell on the periosteal layer in the clinoid space. The last step involves centrally hollowing out the dense cortical bone in the center of the ACP (Fig. 3A). During this procedure, the surgeon must be aware of the relative posi-
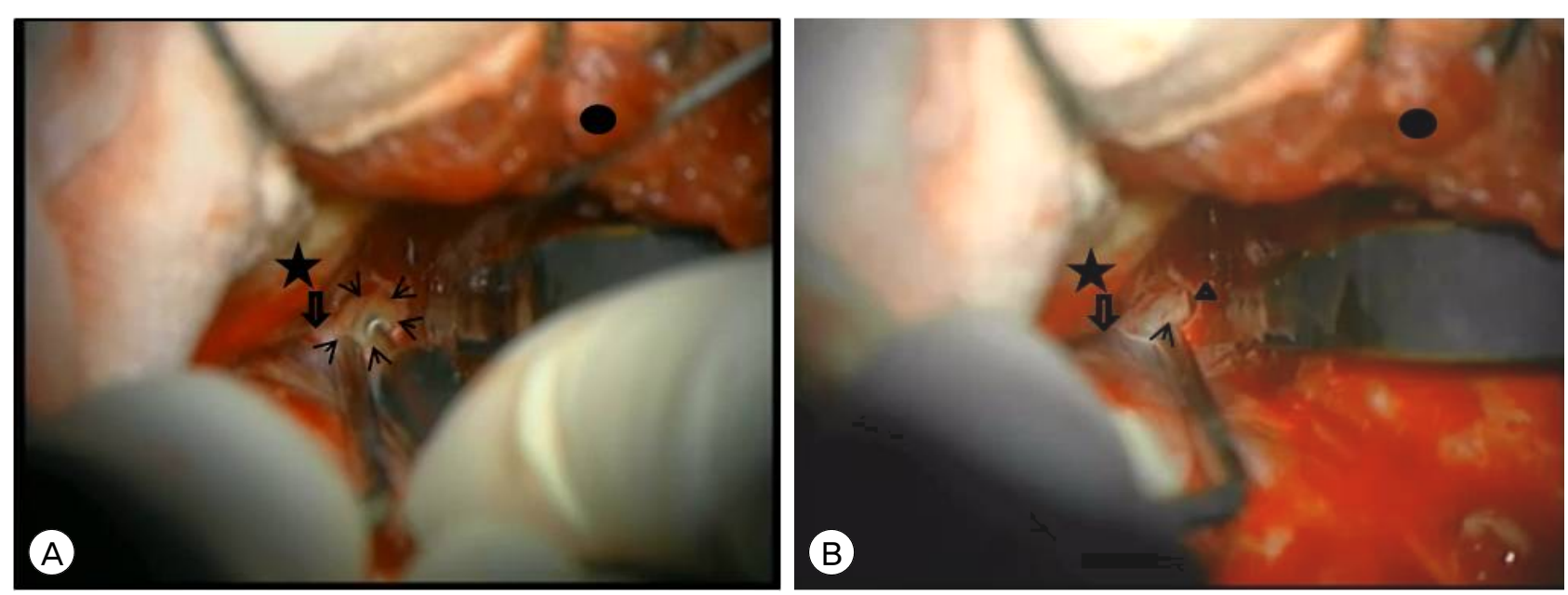

Fig. 3. Intraoperative photography of the anterior clinoid process's centrally shelled or hollowed-out area (The green area of the Fig 1). The outer two-thirds of sphenoid ridge (Asterisk) and the entire lesser wing (Open arrow) are drilled. The temporalis muscle (Dark circle) is retracted red inferiorly. (A) The apex of the ACP is removed using a diamond burr and constant irrigation in an inside cancellous bone to lateral cortical bone. The cortical bone of the ACP (Closed arrow) is remained like a thinned onion shells on the periosteal layer in the clinoid space. The diamond drill is aimed so that the walls can be easily fractured and circumferentially dissected free of the surrounding dural-fold-canal drilling. (B) Intraoperative photography: The optic canal (Open arrow) is broken using a diamond drill. The optic strut (Arrowhead) is remained between the opened clinoid space and the optic canal. The optic strut is dissected from the optic sheath and cleared away using a micropunch. 
tions of the optic nerve, the carotid artery, and the oculomotor nerve with reference to the ACP. It is withdrawn using a microdissector to expose the clinoid space (Fig. 1, green area). The optic canal is broken using a diamond drill. Last, the optic strut remains between the opened clinoid space and the optic canal. The optic strut is dissected from the optic sheath and cleared away using a micropunch (Fig. 3B). Bleeding is easily controlled with bone wax and hemostatic material such as gellfoam.

\section{Dural opening and intradural procedure}

The dura is opened with a semicircular incision extending from the floor of the temporal base at the posterior-inferior aspect of the exposure to the floor of the frontal base at the anterior-inferior aspect exposure. The dura covering the anterior clinoid can be excised to extend the view. We do not need an extended dural incision along the ACP. Wide splitting of the sylvian fissure allows easier retraction of the brain, but this is not done routinely. We can dissect the aneurysm from the carotid artery and optic nerve with a blunt dissector and micro scissors. The clipping techniques of the aneurysms differ depending on the anatomical situation, the shape, and size of the aneurysm.

\section{Closure}

At the end of the clipping, the intradural space is filled with warm saline. The dural incision is closed with prolene 4-0 continuous sutures. The dural around the anterior clinoid leaves covering the medial sphenoid wing are then closed primarily, followed by a watertight closure of the more superficial dural opening. The bone flap is fixed with titanium miniplates and screws. The bone flap should be fixed tightly to achieve optimal cosmetic results. Biopore ${ }^{\mathrm{TM}}$ burr hole covers are thus used on the burr hole site or skull defect area. After the final meticulous hemostasis, the muscle and subcutaneous layers are closed layer by layer.

\section{Case}

\section{Illustrative case 1}

A 46-year-old female presented with an incidental right paraclinoid aneurysm. The $8.66 \times 4.81-\mathrm{mm}$ aneurysm was located at the ophthalmic segment of the ICA, anteriorly and superiorly directed. The preoperative axial T2 MRI scans showed a signal void lesion near the right optic nerve. The images obtained from the digital subtraction angiography of the right carotid artery showed that the saccular aneurysm was directed medial-superiorly and compressed the right optic nerve superolateraly (Fig. 4A). The patient experienced dizziness and diplopia. Her visual-field examination showed right inferior quadrantanopia. A decision was thus made to clip the aneurysm through decompression of the optic nerve instead of coil embolization. The 2-dimensional (2D) CT angiography
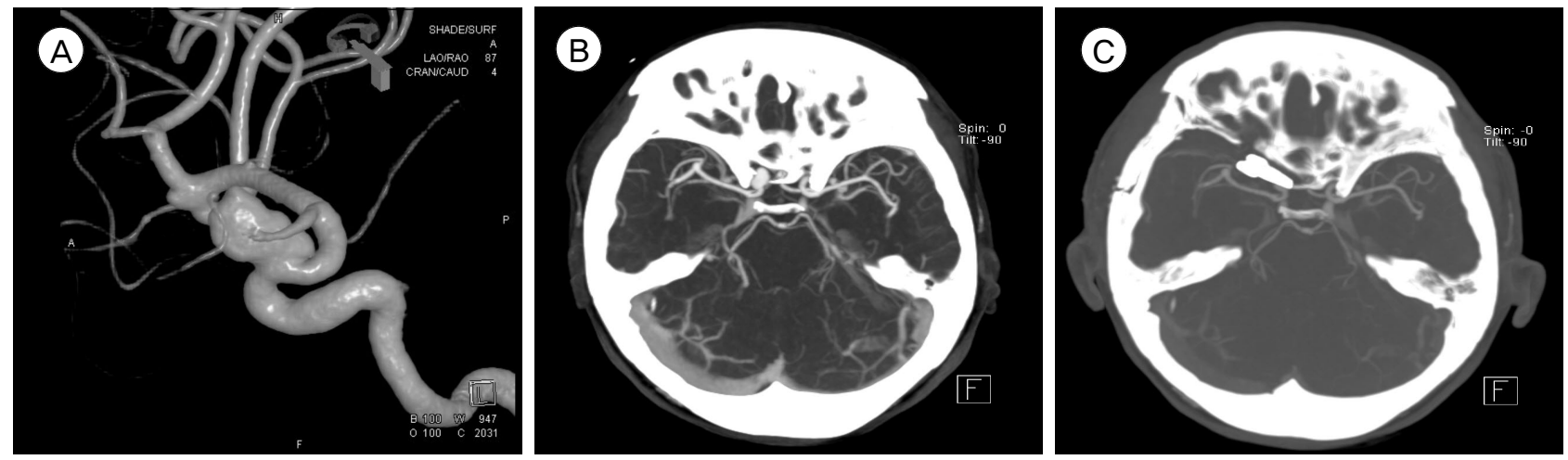

Fig. 4. Case 1: This 46-year-old woman presented with dizziness and diplopia. Her visual-field examination showed right inferior quadrantanopia. (A) Digital subtraction angiography of the right carotid artery shows that the aneurysm is directed superomedially. (B) 2-dimensional (2D) CT angiography shows that the anterior clinoid process (ACP) is an obstacle to the clipping of an aneurysm. (C) Postoperative 2D CT angiography shows that the entire ACP was removed and the aneurysm was clipped with 2 15-mm straight clips. 
showed that the ACP was an obstacle to clipping the aneurysm (Fig. 4B). The aneurysm clipping was finished without complications. The postoperative 2D CT angiography scan shows that the entire ACP was removed, and the aneurysm was clipped with $215-\mathrm{mm}$ straight clips (Fig. 4C). Her visual field was much improved at an 8 months postoperative exam.

\section{Illustrative case 2}

A 42-year-old female presented with sudden-onset severe headache. The CT scan showed right-frontal-base hemorrhage. The 2D angiography showed that the aneurysm was situated in the site of the hemorrhage. The ACP was an obstacle to clipping the aneurysm (Fig. 5A). The 3D CT angiography showed the paraclinoid internal carotid artery aneurysm directed to superolateral side (Fig. 5B). A decision was made to clip the aneurysm through hematoma evacuation. The postoperative CT scan showed that the entire ACP was removed, and the aneurysm was clipped with 2 15-mm curved clips (Fig. 5C).

\section{DISCUSSION}

The lesser wing of the sphenoid presents a sharp posterior border, the sphenoid ridge, which over- hangs the middle cranial fossa and projects into the lateral sulcus of the cerebral hemisphere. It ends medially in the ACP, which attaches to a fold of the dura called tentorium cerebelli, ${ }^{4}$ the medial side of the tentorium with the periclinoid folds, the inter-clinoid folds and the falciform ligament connecting from the $\mathrm{ACP}$ to the planum sphenoidale wrapping up the optic canal roof.5) The ICA entering the cavernous sinus forms the anterior loop between the proximal and distal rings. The ICA, the proximal dural ring at the height of the ACP, becomes the clinoid segment. The ophthalmic segment extends above the distal dural ring. It is continuous to the falciform ligament, to the segment of the posterior communicating artery. The clipping of this paraclinoid segment aneurysm does not have sufficient space for the clipping of the aneurysm neck and proximal control, and that is all essential to remove the apex of the ACP and to open the falciform ligament lateral to the clinoid process. In addition, for clipping large paraclinoid aneurysms, for which it is necessary to open the external dural ring and to mobilize the clinoidal segment of the ICA, total $\mathrm{AC}$ is generally required.

The removal of the ACP provides a better field of the structures in and around the optic nerve, the ICA. $\mathrm{AC}$ and optic-sheath opening significantly increases
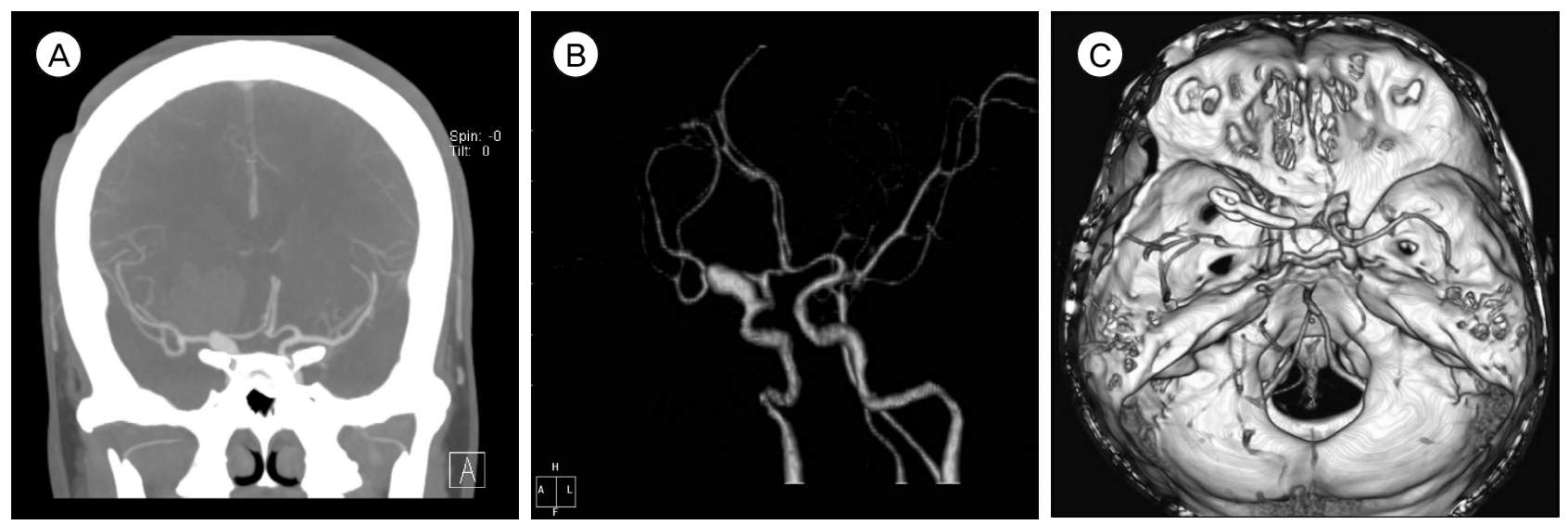

Fig. 5. Case 2: This 42-year-old woman presented with sudden severe headache. (A) The preoperative axial computed tomography (CT) scan shows right-frontal-base hemorrhage. Note that the aneurysm is situated at the site of the hemorrhage. The anterior clinoid process (ACP) is an obstacle to the clipping of an aneurysm. (B) The 3-dimensional (3D) CT angiography shows the superolateral side of the paraclinoid internal carotid artery aneurysm. (C) The postoperative CT scan 3D reconstruction skull image shows that the entire ACP was removed and that the aneurysm was clipped with 2 15-mm curved clips. 
the exposure and mobilization of the optic nerve and ICA as well as 3 to 4 fold expansion of the opticocarotid triangle width.

Yasargil et al. described the pterional approach and intradural AC for the clipping of carotid-ophthalmic aneurysms. ${ }^{10)}$ The pterional approach offers a familiar visualization for most surgeons operating in circle of Willis. Complete splitting of the sylvian fissure makes it easier to identify the proper anatomy of the lesion that may be an obstacle in the initial approach. At that point intradural AC is done. The benefit of intradural $\mathrm{AC}$ is the early exposure of the lesion and tailored ACP resection. If an unintended event occurs during surgery, such as the rupture of an aneurysm, most vascular surgeons will feel more able to control that event.

Dolenc introduced the EAC procedure as part of an extensive transcavernous approach with the opening of the optic sheath in 1985. ${ }^{2)}$ The advantage of EAC is minimal brain retraction. EAC will leave the dura intact when it is frequently opened later in the operation. Thus, the dura mater provides a natural barrier protecting the neurovascular drill burr, and protects the subarachnoid space to the bone ducts. The disadvantage is the risk of injuring the neurovascular structures passing through the SOF during dissecting. ${ }^{12)}$

We favor extradural anterior clinoidectomy for the clipping of paraclinoid aneurysm due to this procedure is done by the second operator. The first operator focuses on the intradural procedure with clipping of the aneurysm.

AC-shaped dural incision is enough to clip of a paraclinoid aneurysm. An additional dural incision parallel to the sylvian fissure is needs to open the distal dural ring for proximal control. We do not need this incision only for proximal control, but we think opening of the distal dural ring for proximal control of the ruptured aneurysm is so risky. In that case we propose to open cervical ICA.

The described major complications related to AC in the modern series include postoperative cerebrospinal fluid leakage, damage to the optic nerve in the form of visual-field deficits due to direct neuronal damage or ischemic injury, oculomotor palsy, and intraoperative aneurysm rupture. ${ }^{16) 9911)}$ Aneurysm rupture during AC is rarely reported. ${ }^{8)}$

The illustrative cases of paraclinoid aneurysm demonstrate the lack of complications. With understanding of the bony anatomy and the dura space around the ACP, EAC can be performed easily.

\section{CONCLUSION}

The authors' experience reviewed in the present literature demonstrates that AC is practicable for clipping paraclinoid aneurysms. The patients were treated with pterional craniotomy and EAC with clipping of paraclinoid aneurysm, and good outcomes were achieved. EAC is also recommended for clipping paraclinoid aneurysms.

\section{REFERENCES}

1. Chi J, Sughrue M, Kunwar S, Lawton M. The "yo-yo" technique to prevent cerebrospinal fluid rhinorrhea after anterior clinoidectomy for proximal internal carotid artery aneurysms. Neurosurgery. 2006 Jul;59(1 Suppl 1):ONS101-7; discussion ONS101-7.

2. Dolenc VV. A combined epi- and subdural direct approach to carotid-ophthalmic artery aneurysms. J Neurosurg. 1985 May;62(5):667-72.

3. Evans JJ, Hwang YS, Lee JH. Pre-versus post-anterior clinoidectomy measurements of the optic nerve, internal carotid artery, and opticocarotid triangle: A cadaveric morphometric study. Neurosurgery. 2000 Apr;46(4):1018-21; discussion 1021-3.

4. Inoue $\mathrm{T}$, Rhoton AL Jr, Theele D, Barry ME. Surgical approaches to the cavernous sinus: A microsurgical study. Neurosurgery. 1990 Jun;26(6):903-32.

5. Rhoton AL Jr. The anterior and middle cranial base. Neurosurgery. 2002 Oct;51(4 Suppl):S273-S302.

6. Romani R, Elsharkawy A, Laakso A, Kangasniemi M, Hernesniemi J. Complications of anterior clinoidectomy through lateral supraorbital approach. World Neurosurg. 2012 May-Jun;77(5-6):698-703.

7. Romani R, Elsharkawy A, Laakso A, Kangasniemi M, Hernesniemi J. Tailored anterior clinoidectomy through the lateral supraorbital approach: Experience with 82 consecutive patients. World Neurosurg. 2012 Mar-Apr;77(3-4):512-7.

8. Romani R, Kivisaari R, Celik Or, Niemela M, Perra G, 
Hernesniemi J. Repair of an alarming intraoperative intracavernous carotid artery tear with anastoclips: Technical case report. Neurosurgery. 2009 Nov;65(5):E998-9; discussion E999.

9. Son HE, Park MS, Kim SM, Jung SS, Park KS, Chung SY. The avoidance of microsurgical complications in the extradural anterior clinoidectomy to paraclinoid aneurysms. J Korean Neurosurg Soc. 2010 Sep;48(3):199-206.

10. Yasargil MG, Gasser JC, Hodosh RM, Rankin TV. Carotid-ophthalmic aneurysms: Direct microsurgical approach.
Surg Neurol. 1977 Sep;8(3):155-65.

11. Yonekawa $Y$, Ogata N, Imhof HG, Olivecrona $M$, Strommer K, Kwak TE, et al. Selective extradural anterior clinoidectomy for supra- and parasellar processes. Technical note. J Neurosurg. 1997 Oct;87(4):636-42.

12. Yoon BH, Kim HK, Park MS, Kim SM, Chung SY, Lanzino G. Meningeal layers around anterior clinoid process as a delicate area in extradural anterior clinoidectomy: Anatomical and clinical study. J Korean Neurosurg Soc. 2012 Oct;52(4):391-5. 